\title{
Studying human immunology and immunopathology in humanized mice transplanted with human lymphoid tissues and immune cells
}

\author{
Cellular \& Molecular Immunology (2012) 9, 191-192; doi:10.1038/cmi.2012.4; published online 7 May 2012
}

$\mathrm{T}$ he major bottleneck of understanding human immunology and immunopathology is the lack of relevant animal models. A robust small animal model for hypothesis testing and mechanistic research on human immunology is needed and should meet the following criteria. First, it has well-studied hematolymphoid organs and target cells which are human. Second, human immune pathology including infection diseases can be modeled in vivo. Third, it is genetically inbred and can be manipulated via immunological, genetic and pharmacological means. The immunodeficient mouse transplanted with human immune organs and cells is the obvious candidate. A number of human-mouse chimeric models were developed in the late 1980s, but with only limited success, due to a lack of significant human immune responses in the SCID-hu Thy/Liv mouse ${ }^{1,2}$ or selective engraftment of xeno-reactive human T cells in the hu-PBL-SCID mouse. $^{3,4}$

New mouse models with functional human immune organs and cells have recently been developed, after the introduction of the IL2R ( or $\gamma c$ ) mutation into Nod-SCID or Rag mutant mice. NodSCID mice with a mutant $\gamma c$ gene (NOG or NSG) have been reported to efficiently engraft human immune cells after transplantation of human $\mathrm{CD}_{3}{ }^{+}$hematopoietic stem cells. The NOG, NSG and Balb/ C Rag2- $\gamma_{c}$ double knockout mice all lack T, B lymphocytes and natural killer cells, and serve as better hosts for engraftment of human cells/ tissues. $^{5-7}$ Remarkably, long-term human T-cell development occurs efficiently in the mouse thymus, and functional human T, B, natural killer and dendritic cells (both $\mathrm{mDC}$ and $\mathrm{pDC}$ ) are readily detected in lymphoid tissues such as bone marrow, thymus, spleen, lymph nodes and peripheral blood. Human T cells developed in the humanized mouse are tolerated to both human and mouse antigens, indicating efficient negative selection by both murine and human APC. Importantly, de novo human B- and T-cell responses are elicited in the humanized mouse by standard immunization (human TT-specific IgG induction) or infection with the human tumor virus EBV (expansion of Epstein-Barr virus-specific CD8 T cells). These Epstein-Barr virus-reactive $\mathrm{T}$ cells respond to Epstein-Barr virus antigens in a human MHC-dependent fashion, suggesting some human $\mathrm{T}$ cells are positively selected by human MHC. In addition, cotransplantation of human hematopoietic stem cells in Nod-SCID-hu Thy/Liv mice

Correspondence: Dr Lishan Su, The University of North Carolina at Chapel Hill, Microbiology and Immunology, 22-048 Lineberger Cancer Center, Chapel Hill, NC 27599, USA.

E-mail: lishan_su@med.unc.edu

Received 8 Feburary 2012; accepted 9 Feburary 2012 also significantly improves engraftment of human immune cells in the peripheral lymphoid organs, including human T cells whose function depends on human HLA. ${ }^{8,9}$

In this special issue, Dr Ito summarizes the history of the recent advancement in humanized mouse models. As the pioneer of the NOG mouse model, Dr Ito presents an update of the humanized mouse models in general and the NOG mouse models in particular. In addition, Dr Ito introduces novel improved NOG mice expressing human cytokines as transgenes, including recent progress in his group that is not published yet. In another review article, Drake et al. summarize their effort to improve the human immune engraftment and functions by expanding human $\mathrm{CD} 34^{+}$hematopoietic stem/progenitor cells, by lentiviral transduction of hematopoietic stem/progenitor cells, and by transient transfection of human cytokines in humanized NSG mice.

In the last few years, such humanized mice have been successfully employed to investigate some of the most critical questions in human immunology. In the review article by Brehm and Shultz, they summarize the history and current status of humanized mouse models for studying human allograft rejection in humanized mice, and discussed the application of these humanized mouse models in human transplant biology. Dr Hu and Dr Yang review the history and current status of humanized mouse models cotransplanted with human hematopoietic stem/progenitor cells and human thymus tissues, and discuss the potential use of these humanized mice in translational biomedical research. Finally, Zhang and Su review the recent advancement of immunopathogenic insights of HIV-1 infection using humanized mouse models. They summarize the history of humanized mice and recent improved models. In addition, they provide an update on recent findings regarding how HIV-1 interacts with human regulatory $\mathrm{T}$ cells and $\mathrm{pDC}$ in humanized mice and how to define the role of human immune effector cells in HIV-1 infection and pathogenesis in vivo.

In summary, the current humanized mouse models have already enabled us to study human immunology and immune pathogenesis in an unprecedented fashion. Human immune development and function in various lymphoid organs in vivo can be investigated in these humanized mouse models. In addition, human immune diseases, including human pathogen infections (HIV-1), tissue/cell graft rejection and graft $v s$. host diseases, have been extensively studied in these models. The recent improvement with human cytokines will further enable us to study improved human immune development and pathogenic immune responses in humanized mice. ${ }^{10}$ In addition, humanization of human immune cells and other human tissues will 
enable a wide range of human pathogens and human diseases to be investigated in vivo. For example, the recent co-engraftment of human immune cells and human liver cells in the immunodeficient mouse enables investigation of hepatitis $\mathrm{C}$ virus infection, human immune responses and human liver diseases. ${ }^{11}$ Other tissues, such as human brain, lung and gut, will also be possible to be cotransplanted with human hematopoietic stem cells. It will also be feasible to generate humanized mouse models with human tissue stem cells derived from human embryonic stem or induced pluripotent stem cells.

Lishan Su

The University of North Carolina at Chapel Hill Microbiology and Immunology 22-048 Lineberger Cancer Center Chapel Hill NC 27599

USA

McCune J, Kaneshima H, Krowka J, Namikawa R, Outzen H, Peault B et al. The SCIDhu mouse: a small animal model for HIV infection and pathogenesis. Annu Rev Immunol 1991; 9: 399-429.
2 McCune JM, Namikawa R, Kaneshima H, Shultz LD, Lieberman M, Weissman IL. The SCID-hu mouse: murine model for the analysis of human hematolymphoid differentiation and function. Science 1988; 241: 1632-1639.

3 Mosier DE, Gulizia RJ, Baird SM, Wilson DB. Transfer of a functional human immune system to mice with severe combined immunodeficiency. Nature 1988; 335: 256259.

4 Tary-Lehmann M, Lehmann PV, Schols D, Roncarolo MG, Saxon A. Anti-SCID mouse reactivity shapes the human $\mathrm{CD} 4^{+} \mathrm{T}$ cell repertoire in hu-PBL-SCID chimeras. J Exp Med 1994; 180: 1817-1827.

5 Ito M, Hiramatsu H, Kobayashi K, Suzue K, Kawahata M, Hioki K et al. NOD/SCID/ mouse: an excellent recipient mouse model for engraftment of human cells. Blood 2002; 100: 3175-3182.

6 Ishikawa F, Yasukawa M, Lyons B, Yoshida S, Miyamoto T, Yoshimoto G et al. Development of functional human blood and immune systems in NOD/SCID/IL2 receptor $\gamma$ chain $^{\text {null }}$ mice. Blood 2005; 106: 1565-1573.

7 Traggiai E, Chicha L, Mazzucchelli L, Bronz L, Piffaretti JC, Lanzavecchia A et al. Development of a human adaptive immune system in cord blood cell-transplanted mice. Science 2004; 304: 104-107.

8 Melkus MW, Estes JD, Padgett-Thomas A, Gatlin J, Denton PW, Othieno FA et al. Humanized mice mount specific adaptive and innate immune responses to EBV and TSST-1. Nat Med 2006; 12: 1316-1322.

9 Yang YG, Sykes M. Xenotransplantation: current status and a perspective on the future. Nat Rev Immunol 2007; 7: 519-531.

10 Willinger T, Rongvaux A, Strowig T, Manz MG, Flavell RA. Improving human hematolymphoid-system mice by cytokine knock-in gene replacement. Trends Immunol 2011; 32: 321-327.

11 Washburn ML, Bility MT, Zhang L, Kovalev GI, Buntzman A, Frelinger JA et al. A humanized mouse model to study hepatitis $C$ virus infection, immune response, and liver disease. Gastroenterology 2011; 140: 1334-1344. 